\title{
Match Running Performance and Positional Demands of a U9 Female Soccer Team Competing in a Parks and Recreation- Sponsored League
}

\author{
Brad H. DeWeese ${ }^{1^{*}}$ (D), Robert W. Sausaman ${ }^{2}$, Matt L. Sams ${ }^{3}$ and Joanne Spalding ${ }^{4}$ \\ ${ }^{1}$ Center of Excellence for Sport Science and Coach Education, East Tennessee State University, USA \\ ${ }^{2}$ Athletic Performance Department, University of Missouri, USA
}

${ }^{3}$ Performance Science, Kansas City Royals, USA

${ }^{4}$ Department of Health Science, Georgia College and State University, USA

* Corresponding author: Brad H DeWeese, Department of Sport, Exercise, Recreation, and Kinesiology, Center of Excellence for Sport Science and Coach Education, East Tennessee State University, Johnson City, TN 37614, USA

\begin{abstract}
The purpose of this study was to describe the match play characteristics of female youth-athletes participating in recreation league U9 soccer via wearable Global Positioning System (GPS) technology. Nine female youth soccer athletes $(8.6 \pm 0.9$ years; all with $3-4$ years of playing experience) were monitored during the study period. The athletes participated in approximately 2.5 hours of soccer-related training per week and two 48-minute $9 \mathrm{v} 9$ matches per week. The athletes were classified as Forwards (FWD), Midfielders (MID), or Defenders (DEF) by the coaching staff at the beginning of the spring season with 139 games analyzed.

Estimated means for total distance, maximum velocity, sprinting, accelerations, and decelerations were 2943 [2715, 3171], 5.57 [5.36, 5.78], 3 [1, 8], 381 [336, 433], and $385[345,430]$, respectively. A statistically significant effect for playing position was found for total distance $(p=0.002)$, while no statistically significant fixed effect was present for maximum velocity $(p=0.301)$. Playing position did not have a statistically significant effect on sprints $(p=0.063)$, whereas statistically significant effects were present for both accelerations $(p=0.003)$ and decelerations $(p<0.001)$.

U9 female soccer players at the recreational level suggest that players cover an average total distance of $2943 \mathrm{~m}$ per match. Current investigation observed U9 female players attained maximal running velocities ranging from 5.17-5.85 $\mathrm{m} / \mathrm{s}$ and experienced nearly 770 change of speed events per match, averaging 381 acceleration events and 385 deceleration events. This information may help sport practitioners develop age-specific training programs with a priority on long-term development.
\end{abstract}

\section{Keywords}

Youth soccer, Female, GPS

\section{Introduction}

Within the United States, soccer ranks third in participation rates with an estimated 11.9 million total registrants [1]. Moreover, the most recent data demonstrates that approximately 2.3 million children between the ages of 6-12 play the sport of soccer on a regular basis, equating to $37 \%$ of all youth soccer participants. Surprisingly, while this segment of the sporting population serves as the second-largest pool for athletes under the age of 19 [2], little to no scholarly literature exists on youth-soccer match demands within the United States.

This gap in the literature comes as a disadvantage for domestic youth-soccer program providers and global proponents of Long-Term Athlete Development (LTAD) alike. Long-term athlete development models promote gradual, systematic, and progressive exposure to training loads that is congruent to maturational stages. The goal of any LTAD approach is for young athletes to attain optimal development of their skills and physical abilities whilst avoiding the consequences of excessive or inappropriate training [3]. In other words, LTAD deals with the construction of a longitudinal curriculum

Citation: DeWeese BH, Sausaman RW, Sams ML, Spalding J (2020) Match Running Performance and Positional Demands of a U9 Female Soccer Team Competing in a Parks and Recreation-Sponsored League. Int J Sports Exerc Med 6:179. doi.org/10.23937/2469-5718/1510179

Accepted: December 17, 2020; Published: December 19, 2020

Copyright: (C) 2020 DeWeese $\mathrm{BH}$, et al. This is an open-access article distributed under the terms of the Creative Commons Attribution License, which permits unrestricted use, distribution, and reproduction in any medium, provided the original author and source are credited. 
that paves the way from the playground to the podium. To successfully construct sequenced models, however, knowledge of match demands across the developmental spectrum is required.

Unfortunately, scientific literature investigating the physical and physiological characteristics and match demands of female soccer is limited in comparison to the body of research available for male players [4]. In fact, the only literature available on female athletes has been published at the collegiate and professional levels. For example, Sausaman and colleagues [5] examined match play for female athletes at the National Collegiate Athletic Association Division I level and found average distances of approximately $9.5 \mathrm{~km}$ per match. Similarly, research on sub-elite and elite female athletes [6-8]. Has demonstrated these athletes cover approximately 8-11 km per match. In either case, these values tend to be at the lower end of published data for male athletes [4]. Given the dearth of information available for female youth-athletes, practitioners may incorrectly turn to data published on male athletes for insight. This approach is problematic, however, for two reasons: 1) Much of the male youth-athlete research has been conducted in international academy systems [9-15] and 2) Extrapolating male match demands to female athletes may lead to overestimation of match play physical requirements given the disparities between male and female match demands as mentioned above. Simply put, these underlying factors limit the applicability and relevance to the development of female soccer within the United States, where $42.3 \%$ of athletes are between the ages of 6 and 12 [1] and compete within community-based recreation leagues and regional club-systems.

Couched in the above discussion on LTAD, a comprehensive understanding of match demands across age ranges and competitive levels is required to enhance the developmental pipeline for female soccer athletes. Therefore, the purpose of this study was to describe the match play characteristics of female youth-athletes participating in recreation league U9 soccer. This study aimed to examine the athletes' match demands via wearable Global Positioning System (GPS) technology, with the hope of linking data observed at the recreational level with the data that have been published within the rest of the youth development system.

\section{Methods}

\section{Experimental approach to the problem}

This observational study was designed to describe the match demands of parks and recreation sponsored league U9 female soccer. The match data were collected over two consecutive seasons (spring and fall) via wearable GPS units. The athletes were classified as forwards, midfielders, or defenders. Mixed-effects models were fit to characterize the match play characteristics of each position group.

\section{Subjects}

Nine female youth soccer athletes $(8.6 \pm 0.9$ years; all with 3-4 years of playing experience) were monitored during the study period. The athletes participated in approximately 2.5 hours of soccer-related training per week and two 48-minute 9v9 matches per week. The athletes were classified as Forwards (FWD), Midfielders (MID), or Defenders (DEF) by the coaching staff at the beginning of the spring season. Returning athletes remained at these positions during the fall season, while new athletes were assigned to a position group prior to the first match. University institutional review board approval was granted for the study. Prior to study enrollment, the athletes and their parents/guardians were informed about the purposes of the data collection, and all nine athletes' guardians gave consent to the collection and analysis of their children's data. All athletes and guardians were assured involvement was voluntary and that they were free to withdraw at any time.

\section{Procedures}

Soccer match play: All matches were played with modified versions of the rules defined by US Soccer. Specifically, all matches were 9v9 (8 outfield players and a designated goalkeeper), were contested on a 35 $\times 55$ yard pitch, and lasted approximately 48 minutes. The matches were split into 12-minute quarters. Quarters were separated by a two-minute break, whereas half-time lasted approximately five minutes. In order to promote and ensure equitable participation, U9 soccer is allowed unlimited substitutions--athletes are able to interchange with teammates during any stoppage of play. In addition, the league's rules dictated each child must play a minimum of 12 minutes per game. Therefore, a "complete" game was defined as participation in 36 minutes of game play for a specified match.

Data collection and processing procedures: Prior to beginning the team warm-up, the athletes were fitted with GPS units (STATSports Apex, Newry, Northern Ireland) sampling at $10 \mathrm{~Hz}$. In addition to their GPS capabilities, each unit contains a triaxial accelerometer sampling at $952 \mathrm{~Hz}$. Data investigating the validity and reliability of these units are published elsewhere [16,17].

The units were placed on the upper back (i.e., between the shoulder blades) of each athlete using custom-made, tight-fitting vests to reduce movement artifacts. The devices were switched on 15-20 minutes before the team warm-up and were immediately switched off at the end of match play [18]. As mentioned previously, league rules influenced the substitution patterns employed by the coaching staff. When athletes were not on the pitch, their units were "benched"; benching units prevented data from being added to the aggregated statistics at the end of a match. Start and stop times for each quarter, along with benchings, were set by the coaching staff concurrent with match play. To ensure 
data integrity, however, all match files were retroactively examined by the researchers and start-stop times and benchings were adjusted as necessary. Initial processing was performed in manufacturer-provided software (STATSports Apex Pro Series) prior to the data being exported for further processing and analysis.

Movement profiles were created to describe match demands and to monitor player progress and development. Assessed movement profile variables included total distance; maximum velocity achieved; and counts of sprints, accelerations, and decelerations in a given match. To be considered a sprint, a run had to cross a threshold of $80 \%$ of an athlete's maximum velocity for a minimum of one second. Maximum velocities were set at the beginning of each season via a flying $10 \mathrm{~m}$ sprint test, but an athlete's value was adjusted if a higher velocity was observed during match play. Similarly, events were registered as accelerations or decelerations if the accelerometer registered an acceleration exceeding \pm $3 \mathrm{~m} \cdot \mathrm{s}^{-2}$. Sprint and acceleration thresholds were set via manufacturer recommendations. Counts for the number of sprints, accelerations, and decelerations were reported as these are among the most common variables of interest for researchers and practitioners $[6,17]$.

\section{Statistical analysis}

All additional processing and statistical analyses were performed in R (version 3.6.2) [19]. Match summary statistics (e.g., minutes played, total distance) were calculated for each match appearance for each athlete, and matches with less than 36 minutes played were filtered from the dataset. Post-filtering, 139 games (mean games per athlete: 15; range:4 -24) were available for the model fitting process.

A series of mixed-effects models were fit via the Ime4 package (version 1.1-23) [20] to assess differences between playing positions. In the case of total distance and maximum velocity, Linear Mixed-Effects Models (LMM) were fit. For counts of sprints, accelerations, and decelerations, however, Generalized Linear Mixed-Effects Models (GLMM) were specified with a Poisson distribution and log-link function. In either case, the models were fit with the respective dependent variable, a fixed effect for playing position, and crossed random-intercept effects for athlete and match. Model assumptions for LMM were checked via visual inspection of quantile-quantile plots and fitted-vs-residuals plots, whereas GLMM were assessed via the DHARMa package (version 0.3.1) [21]. No violations of model assumptions were detected for any dependent variable. F-statistics were calculated via the Kenward-Roger approximation to assess the statistical significance of playing position for total distance and maximum velocity, while chi-square tests were performed to assess the effect of playing position on sprints, accelerations, and decelerations. Pairwise comparisons were planned for all models, regardless of statistical significance.

Estimation of marginal means, pairwise comparisons with a Tukey-Kraemer adjustment, and effect size calculations were carried out via the emmeans package (version 1.4.5) [22]. Effect Sizes (ESs) were calculated for total distance and maximum velocity only. Based on recommendations from the literature, Cohen's $d$ was calculated as the mean difference between positions divided by the square root (sqrt) of the respective model's pooled variance $[23,24]$. For the remaining variables, the log estimates were back-transformed and the resulting ratio represented the difference between groups. Data are presented as their model-derived estimates and 95\% confidence intervals ( $95 \% \mathrm{Cls}$ ). Pairwise comparisons for continuous data are presented as the estimate $\pm 95 \% \mathrm{Cl}$, whereas comparisons for count data are the estimate $x$ $95 \% \mathrm{Cl}^{ \pm 1}$. Effect sizes are presented as the estimate.

\section{Results}

Estimated match averages for each dependent variable are visualized in Table 1 (group-level) and Figure 1 (individual-level). Overall estimated means [95\% Cl] for total distance $(\mathrm{m})$, maximum velocity $\left(\mathrm{m} \cdot \mathrm{s}^{-1}\right)$, sprinting (\#), accelerations (\#), and decelerations (\#) were 2943 [2715, 3171] , 5.57 [5.36, 5.78], 3 [1, 8], 381 [336, 433], and 385 [345, 430], respectively. A statistically significant effect for playing position was found for total distance $(F[2,5.473]=22.207, p=0.002$, sqrt pooled variance $=670.817)$, while no statistically significant fixed effect was present for maximum velocity $(F[2,5.966]$ $=1.478, p=0.301$, sqrt pooled variance $=0.432)($ Table 2). Playing position did not have a statistically significant effect on sprints $\left(\chi^{2}=5.526, p=0.063\right)$, whereas statistically significant effects were present for both accelerations $\left(\chi^{2}=11.392, p=0.003\right)$ and decelerations $\left(\chi^{2}=\right.$

Table 1: Model-derived means for assessed movement characteristics.

\begin{tabular}{|l|l|l|l|}
\hline & FWD & MID & DEF \\
\hline Distance (m) & $3029[2728,3330]$ & $3506[3155,3858]$ & $2293[1972,2614]$ \\
\hline MaxVelo (m/s) & $5.44[5.08,5.80]$ & $5.49[5.12,5.86]$ & $5.77[5.41,6.14]$ \\
\hline Sprints (\#) & $9[2,36]$ & $5[1,21]$ & $1[0,4]$ \\
\hline Accelerations (\#) & $413[341,500]$ & $450[371,545]$ & $299[247,362]$ \\
\hline Decelerations(\#) & $412[350,485]$ & $454[385,535]$ & $305[259,359]$ \\
\hline
\end{tabular}

FWD, forwards; MID, midfielders; DEF, defenders; MaxVelo, maximum velocity.

Data are mean $[95 \% \mathrm{Cl}] .95 \% \mathrm{Cl}$ for count data are asymptotic. 

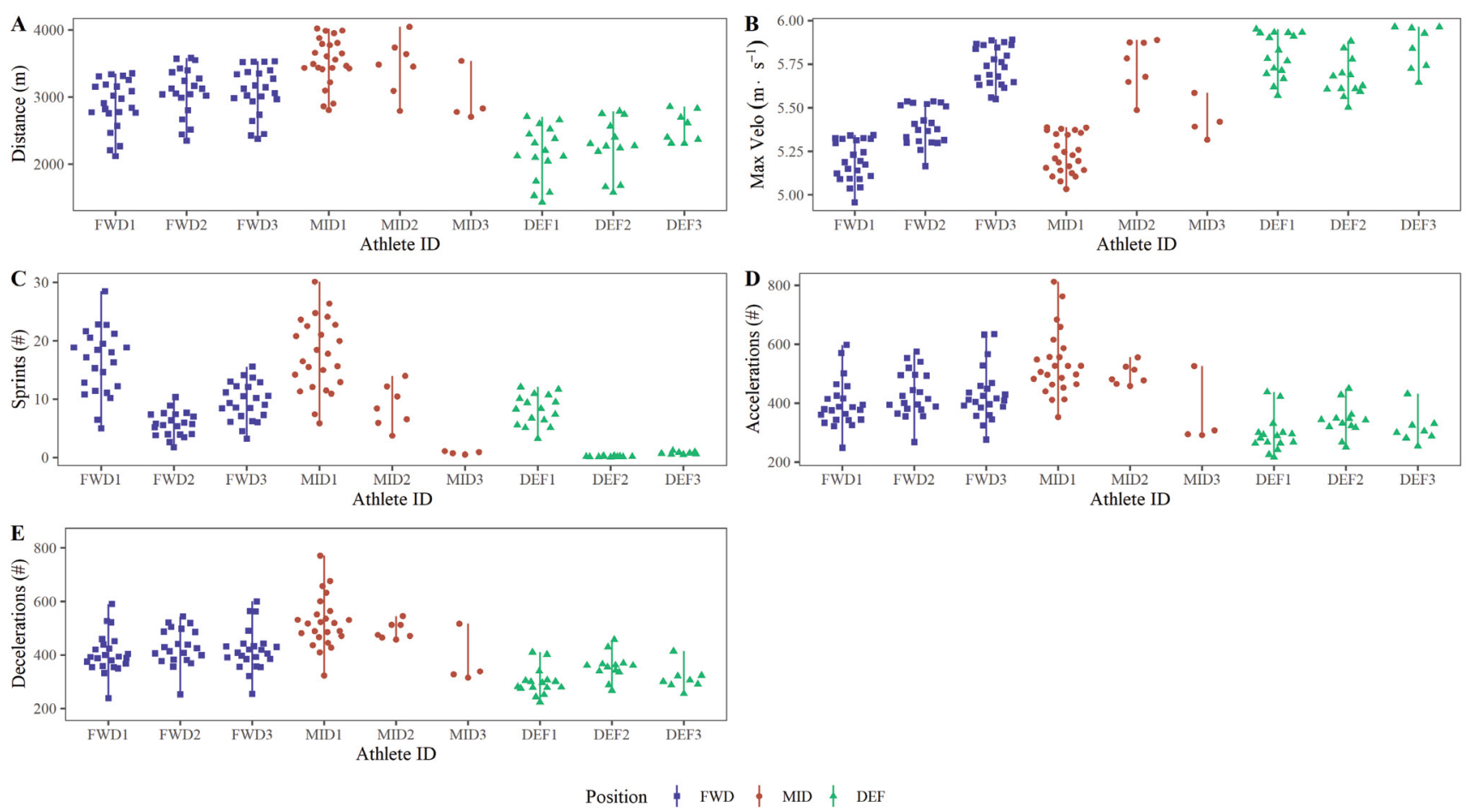

Figure 1: Model predictions for individual athletes. Predictions account for both fixed and random effects. FWD, forward; MID, midfielder; DEF, defender.

Table 2: Pairwise comparisons for assessed movement characteristics.

\begin{tabular}{|l|l|l|l|l|}
\hline & FWD - MID & FWD - DEF & MID - DEF & Difference \\
\hline Continuous Variables & & & & \\
\hline Distance $(\mathrm{m})$ & $-477[-1034,79],-0.71$ & $736[217,1255], 1.10$ & $1213[657,1769], 1.81$ & FWD, MID > DEF \\
\hline MaxVelo $(\mathrm{m} / \mathrm{s})$ & $-0.05[-0.69,0.60],-0.11$ & $-0.33[-0.97,0.31],-0.77$ & $-0.28[-0.93,0.36],-0.65$ & ND \\
\hline Count Variables & & & & \\
\hline Sprints (\#) & $1.76[0.16,19.49]$ & $10.65[0.93,122.60]$ & $6.05[0.51,71.6]$ & ND \\
\hline Accel (\#) & $0.92[0.68,1.24]$ & $1.38[1.02,1.86]$ & $1.51[1.12,2.03]$ & FWD, MID $>$ DEF \\
\hline Decel (\#) & $0.91[0.71,1.17]$ & $1.35[1.06,1.74]$ & $1.49[1.16,1.91]$ & FWD, MID $>$ DEF \\
\hline
\end{tabular}

FWD, forwards; MID, midfielders; DEF, defenders; Max Velo, maximum velocity.

Difference column represents statistically significant contrasts $(p<0.05)$. ND, no differences.

Continuous variable contrasts are mean difference [95\% Cl], ES.

Count variable contrasts are ratio difference $[95 \% \mathrm{Cl}]$. Ratio $95 \% \mathrm{Cl}$ are asymptotic.

15.202, $p<0.001$ ) (Table 2).

\section{Discussion}

The purpose of this investigation was to describe the match play characteristics of female youth-athletes participating in parks and recreation league U9 soccer. The analysis revealed different movement profiles between playing positions, with FWD and MID generally covering greater distance and performing more accelerative and decelerative actions compared to DEF.

This investigation into the physical demands of match play for U9 female soccer players at the recreational level suggests that players cover an average total distance of $2943 \mathrm{~m}$ per match. While comparisons are limited due to a dearth of literature, this total distance is above the $2229 \mathrm{~m}$ traveled by the male U8 national team youth soccer players observed by Bellistri and colleagues [25]. However, this volume of movement appears to be lower than the $3541 \mathrm{~m}$ displaced by the male U10 athletes of the same investigation. Furthermore, this is significantly less than the observations published by Goto, et al. (2015) and Saward, et al. (2016), who reported a range of 4300-5390 for professional academy U9 and U10 male soccer players.

Collectively, the differences found between the available research and the present study can likely be explained by several factors that are inherently shaped by age-related changes, the level of competition, and league-specific rules. First, Saward, et al. (2016) demonstrated a steady increase of total distance from the ages of 8 to 18 , which eventually leveled off at 17.7 years. Secondly, other research has established that inter- 
national-class players experience greater match demands in comparison to their professional counterparts $[13,14,26]$. Therefore, one can reasonably infer a similar difference exists between competitive levels across all age ranges. In addition to the effect of age and competitive level, the rules governing match play between academy-sponsored youth soccer and American recreation soccer are distinctly different. For instance, the duration of matches observed within this current study are at least 12 minutes less than competitions reported by Saward (2016) and Goto (2015). Further, youth soccer competed at the recreation level places a premium on equitable participation, which limits the ability to accurately compare competitive match demands against academy players. Such differences between age groups and league structure (level, rules, match duration) are critical to identify because of the significance such information plays in creating a seamless progression throughout the developmental pathway.

Unlike previous studies, which have not reported on maximal running velocities or acceleration metrics, the current investigation observed U9 female players attained maximal running velocities greater than $5.5 \mathrm{~m} \cdot \mathrm{s}$ 1 , performed at least 3 sprint efforts, and experienced approximately 770 change of speed events--split evenly between 381 accelerations and 385 decelerations--per match. While technical proficiency is a requisite for sporting success, soccer coaches and practitioners often prioritize it while neglecting to consider the strategic development of physical competencies [27]. In fact, research suggests that sprinting and the ability to quickly accelerate or decelerate are not only fundamental characteristics required in soccer, but are pivotal attributes affecting match outcomes as well as differentiating players between competitive levels. For example, players with superior acceleration abilities have the potential to be quicker to the ball or open more space over opponents, leading to greater ability to score goals or defend against a goal being scored $[13,28,29]$. Taken together, it seems intuitive that developing physical qualities such as acceleration/deceleration, maximal sprint speed, and explosive strength would benefit players. Therefore, placing a greater emphasis on these qualities, particularly in developmental stages, will likely enhance player abilities to cope with increasing matched demands and the growing reliance on sprint speed across age groups [27] and spanning between competitive levels $[26,30,31]$. Similarly, as women's soccer continues to evolve [27,31], locomotive technique and speed will continue to play a vital role in tolerating hastened match scenarios while perhaps reducing the likelihood of injury. In short, the available literature and present findings provide merit for the adoption and implementation of a formal sprinting and change of direction curriculum corollary to technical, "on the ball" skill work within the LTAD process. Namely, a training agenda that introduces sound running technique which progresses seam- lessly through the phases of maturation. While further study would be needed, adding robustness to a youth soccer player's physical literacy training plan may raise the ceiling for performance potential downstream, particularly if the plan emphasizes proper acceleration and deceleration technique.

Despite the limited sample size, some of the present data align with previously published observations in male athletes. For instance, MID tended to accumulate more total distance per game when compared to FWD and DEF, which is similar to the observations of Buchheit, et al. and Kovács, et al. [7,28]. This is likely due to their box-to-box responsibilities and role linking DEF and FWD. Contrary to previous research in mature soccer populations [11,31,32], defenders tended to demonstrate the highest velocities within this study, which highlight the differing physical demands that are incurred by playing positions. This finding may have been a function of the team's competitive success: across the two seasons, the team accumulated a win-loss-draw record of 23-1-1 with a goal differential of 169-22. Utilizing a 3-2-3 formation and playing primarily in the middle and attacking thirds of the pitch may have constrained the space available for 3 ATT to achieve higher running speeds, while also providing DEF more space to develop speed in the event of a counterattack. Examining Figure 1 , however, it is important to note the Variability of the athletes' movement profiles, which may be a function of individual differences between athletes. Particularly at younger ages, such differences may be the result of numerous factors including physical maturation status, technical proficiency, tactical awareness, superior motor skills, and coordination [33-35].

This latter point brings up an important discussion of the limitations of the present study. Namely, the data were collected from a single cohort of U9 soccer athletes all competing on the same team. League equitability rules, team playstyle, and the vast competitive difference between the present athletes and the rest of the league should lead to cautious generalized extrapolation of the findings. Similarly, the small sample size only left us the power to detect relatively large differences between positions, a problem that is inherent--but not unique--to sport science [23]. Regardless, these findings are an important first step in investigating the youth female soccer LTAD process and reinforce the differences in competitive demands faced by male and female athletes.

In the greater context of the developmental process, practitioners are advised to consider the variability in physical and technical maturities in youth athletes and to remember the role of sport for this demographic. Particularly, sport plays a vital role in exposing young athletes to a myriad of movement skills, mental strategies, and social dynamics that will provide value across the lifespan. Therefore, entry into competitive youth sport 
should be characterized by training agendas that stage a safe and gradual exposure to loads necessary for participatory success. Moreover, with nearly $600,000(14 \%)$ of these 6- to 12-year-old participants leaving the sport of soccer over the last three years [2], it appears to be an appropriate time to consider modifications to the youth soccer system within the United States. While cost, travel, and performance anxiety likely contribute to this dropout rate, the lack of accurate training doses resulting from previously unexplored competitive demands are not helpful $[2,36]$. Gaining clarity on U9 female soccer performance measures may equip club directors, recreation coordinators, and coaches with the necessary information to cultivate a long-term developmental plan that is specific to their community and more in-line with the needs of this age group. Furthermore, this insight may allow parents to nurture their children's sporting career through supplemental, multilateral fitness that promotes a seamless transition into seasonal soccer play.

\section{Practical Applications}

The findings of the current investigation broaden our understanding of the physical demands experienced by female youth soccer players during match play. Specifically, these data can inform LTAD models in order to refine training strategies that allow for younger athletes to safely tolerate the match loads typical of U9 soccer. Clarifying match demands at different age groups can aid sport practitioners in developing players as they progress through age groups or are promoted up levels of play and can help avoid training youth players as mini-adults. Instead, these training programs can be designed to build resilient players who are able to meet the demands of the game at their respective age groups. Further, with these data, we can begin to establish normative data relative to sex, age, level, and competitve structure--a critical first step in identifying if and when players demonstrate the requisite running capacity, speed, and skill required to safely progress along the developmental pathway. Finally, the present data suggest that position-specific demands do exist within 9v9 match play, which may assist in the refinement of talent identification protocols.

\section{Funding}

No funding was received for this study.

\section{References}

1. Sport and Fitness Industry Association (2018) Soccer participation in the United States.

2. Aspen Institute (2018) State of Play 2018.

3. Dellal A, Owen A, Wong DP, Krustrup P, van Exsel M, et al. (2012) Technical and physical demands of small vs. large sided games in relation to playing position in elite soccer. Hum Mov Sci 31: 957-969.

4. Martínez-Lagunas V, Niessen M, Hartmann U (2014) Women's football: Player characteristics and demands of the game. Journal of Sport and Health Science 3: 258-272.

5. Sausaman RW, Sams ML, Mizuguchi S, DeWeese BH, Stone MH (2019) The Physical Demands of NCAA Division I Women's College Soccer. JFMK 4: 73.

6. Abbott W, Brickley G, Smeeton N (2017) Positional differences in GPS outputs and perceived exertion during soccer training games and competition. J Strength Cond Res 32: 3222-3231.

7. Buchheit M, Horobeanu C, Mendez-Villanueva A, Simpson BM, Bourdon PC (2011) Effects of age and spa treatment on match running performance over two consecutive games in highly trained young soccer players. J Sports Sci 29: $591-598$.

8. Mujika I, Santisteban J, Impellizzeri F, Castagna C (2009) Fitness determinants of success in men's and women's football. Journal of Sports Sciences 27: 107-114.

9. Atan SA, Foskett, A, Ali A (2016) Motion Analysis of Match Play in New Zealand U13 to U15 Age-Group Soccer Players. J Strength Cond Res 30: 2416-2423.

10. Castagna C, D'Ottavio S, Abt G (2003) Activity profile of young soccer players during actual match play. J Strength Cond Res 17: 775-780.

11. Goto H, Morris J, Nevill ME (2015) Match analysis of U9 and U10 English Premier League Academy Soccer Players using a Global Positioning System: Relevance for Talent Identification and Development. J StrengthCond Res 29: 954-963.

12. Harley JA, Barnes CA, Portas M, Lovell R, Barrett S, et al. (2010) Motion analysis of match-play in elite U12 to U16 age-group soccer players. J Sports Sci 28: 1391-1397.

13. Palucci Vieira LH, Carling C, Barbieri FA, Aquino R, Santiago PRP (2019) Match Running Performance in Young Soccer Players: A Systematic Review. Sports Med 49: 289-318.

14. Reilly T, Williams AM, Nevill A, Franks A (2000) A multidisciplinary approach to talent identi $\circledast$ cation in soccer. $J$ Sports Sci 18: 695-702.

15. Saward C, Morris JG, Nevill ME, Sunderland C (2009) Longitudinal development of match-running performance in elite male youth soccer players. Scand J Med Sci Sports 4.

16. Beato M, Coratella G, Stiff A, lacono AD (2018) The validity and between-unit variability of GNSS units (STATSports Apex 10 and $18 \mathrm{~Hz}$ ) for measuring distance and peak speed in team sports. Front Physiol 9: 1288.

17. Varley MC, Fairweather IH, Aughey RJ (2012) Validity and reliability of GPS for measuring instantaneous velocity during acceleration, deceleration, and constant motion. Journal of Sports Sciences 30: 121-127.

18. Duffield R, Reid M, Baker J, Spratford W (2010) Accuracy and reliability of GPS devices for measurement of movement patterns in confined spaces for court-based sports. Journal of Science and Medicine in Sport 13: 523-525.

19. $R$ Core Team (2019) R: A language and environment for statistical computing. Vienna, Austria: R Foundation for Statistical Computing.

20. Bates D, Mächler M, Bolker B, Walker S (2015) Fitting linear mixed-effects models using Ime4. J Stat Soft 67.

21. Hartig F (2020) DHARMa: Residual diagnostics for hierarchical (multi-level / mixed) regression models.

22. Lenth R (2020) emmeans: Estimated marginal means, aka least-squares means. 
23. Brysbaert M, Stevens M (2018) Power analysis and effect size in mixed effects models: A tutorial. J Cogn 1: 9.

24. Judd CM, Westfall J, Kenny DA (2017) Experiments with More Than One Random Factor: Designs, Analytic Models, and Statistical Power. Annu Rev Psychol 68: 601-625.

25. Bellistri G, Marzorati M, Sodero L, Sforza C, Bradley PS, et al. (2017) Match running performance and physical capacity profiles of U8 and U10 soccer players. Sport Sci Health 13: $273-280$.

26. Mohr M, Krustrup P, Andersson H, Kirkendal D, Bangsbo $\mathrm{J}$ (2008) Match Activities of Elite Women Soccer Players at Different Performance Levels. J Strength Cond Res 22 : 341-349.

27. Ramos G, Nakamura F, Penna E, Wilke C, Pereira L, et al. (2019) Activity Profiles in U17, U20, and Senior Women's Brazilian National Soccer Teams During International Competitions: Are There Meaningful Differences? J Strength Cond Res 33: 3414-3422.

28. Kovács BM, Malone J, Bognár M, Bali P, Pánics G (2020) Influence of team success, fixture congestion and playing position on physical performance of elite youth soccer players during an international tournament. jhse 16.

29. Ryan D, Lewin C, Forsythe S, McCall A (2018) Developing World-Class Soccer Players: An Example of the Academy Physical Development Program From an English Premier
League Team. Strength and Conditioning Journal 40: 10.

30. Barnes C, Archer D, Hogg B, Bush M, Bradley P (2014) The evolution of physical and technical performance parameters in the English Premier League. Int J Sports Med 35: 10951100.

31. Datson N (2016) An analysis of the physical demands of international female soccer match-play and the physical characteristics of elite players. Doctoral Thesis, Liverpool John Moores University, Liverpool.

32. Mohr M, Krustrup P, Bangsbo J (2003) Match performance of high-standard soccer players with special reference to development of fatigue. J Sports Sci 21: 519-528.

33. Práxedes A, Moreno A, García-González L, Pizarro D, Del Villar F (2017) The Relative Age Effect on Soccer Players in Formative Stages with Different Sport Expertise Levels. J Hum Kin 60: 167-173.

34. Rouissi M, Owen AL, Bragazzi NL, Achour SB, Chamari K (2019) Physical characteristics and strength ratios differentiating startervs. non-starter elite youth soccer players. 6: 8.

35. Smith KL, Weir PL, Till K, Romann M, Cobley S (2018) Relative Age Effects Across and Within Female Sport Contexts: A Systematic Review and Meta-Analysis. Sports Med 48: $1451-1478$.

36. Bangsbo J (2014) Physiological demands of football. 27: 6. 